\title{
Rechtsgeschichte
}

www.rg.mpg.de

http://www.rg-rechtsgeschichte.de/rg15

Zitiervorschlag: Rechtsgeschichte Rg 15 (2009)

$\operatorname{Rg} 15_{2009}$

$207-209$

http://dx.doi.org/10.12946/rg15/207-209

\section{Joachim Eibach}

Der verunstaltete Krieg und die vergessene Kriegserfahrung 


\section{Der verunstaltete Krieg und die vergessene Kriegserfahrung*}

Das Thema Krieg, verstanden als individuelle und kollektive Kriegserfabrung, ist in der deutschen wie auch in der europäischen Erinnerungskultur inhaltlich besetzt durch das Leiden in den beiden Weltkriegen des 20. Jahrhunderts. Als große, stotale` Kriegskatastrophe kommt sonst höchstens noch der Dreißigjährige Krieg in den Blick. Die Ära der Revolutions- und der napoleonischen Kriege zwischen I792 und I 8 I 5 ist dagegen zwar unter verschiedensten Aspekten immer wieder zum Thema geworden, sowohl in der Forschung als auch im Feuilleton. Das Kriegsgeschehen als solches spielte dabei aber eine untergeordnete Rolle. Vielmehr verklärte die borussische Geschichtsschreibung ab der zweiten Hälfte des I9. Jahrhunderts die militärischen Auseinandersetzungen wirkmächtig zum nationalen Aufbruch als >Befreiungskrieg` von französischer Fremdherrschaft. Das deutet der Titel von Ute Planerts umfassender Studie an. Hinzuzufügen ist, dass auch das einflussreiche Transformationstheorem $\mathrm{der}>\mathrm{Mo}$ dernisierung ‘ auf Seiten der, politisch Verwestlichung anstrebenden, Historischen Sozialwissenschaft die Realität des Krieges ausblendete, vielleicht stärker noch als die Alt-Borussen. Indes fiel der Krieg als Phänomen der Erfahrung und Lebenswelt der betroffenen Menschen in beiden Fällen durch das heuristische Maschenwerk von Heldentum bzw. Strukturveränderung. Es kommt nicht von ungefähr, dass der Nahblick auf den Alltag des Krieges als Katastrophe für den Dreißigjährigen Krieg etwas früher rekonstruiert wurde als für die Konflikte um I800. ${ }^{\mathrm{I}}$ Die dominanten Interpretationen in ihrem jeweiligen politischen Kontext erschwerten bislang ein anderes Verständnis der napoleonischen Kriege.

Ute Planert hat diesem verunstalteten und vergessenen Kriegsalltag zwischen I792 und I8I 5 ihre 739 Seiten zählende Habilitationsschrift gewidmet. Sie richtet den Fokus auf die Lebenswelt des Kriegsgeschehens, d. h. die Praxis der Einquartierungen, Durchmärsche, Plünderungen und Requisitionen, gefolgt von Seuchen und wirtschaftlicher Not. Gegenüber dem preußisch-nationalistischen Mythos vom >Befreiungskrieg< will die Autorin einen dreifachen Perspektivenwechsel vornehmen. Sie thematisiert, erstens, das Rheinbund-Deutschland, genauer die sich neu formierenden süddeutschen Territorien Bayern, Württemberg und Baden. Zweitens, versteht sie die Ära der Revolutions- und napoleonischen Kriege vor und nach I806 als eine Einheit. Am wichtigsten aber ist, drittens, der erfahrungsgeschichtliche Ansatz. Ziel ist eine »breit angelegte Erfahrungsgeschichte« (66). Die Kategorie Erfahrung wird dabei kulturhistorisch verstanden als vermittelnde Dimension zwischen Struktur und Subjekt, kontextbezogen, sozial und diskursiv figuriert.

Um das Kriegsgeschehen als Alltag und Wahrnehmung rekonstruieren zu können, wertet Planert "subjektive Zeugnisse" $(56)$ aus, d.h. Selbstzeugnisse der betroffenen Akteurinnen und Akteure. Dadurch wird es möglich, die offizielle Staatspropaganda und die später sich durchsetzende Sichtweise des zunehmend nationalistischen Bürgertums zu konterkarieren. Das Zusammentragen solcher Selbstzeugnisse ist mühselig. Die Autorin fand sie nicht nur in den großen Staatsarchiven, sondern auch in Adels-,

\footnotetext{
Ute Planert, Der Mythos vom Befreiungskrieg. Frankreichs Kriege und der deutsche Süden: Alltag - Wahrnehmung - Deutung I792-I 84I (Krieg in der Geschichte, Bd. 33), Paderborn: Ferdinand Schöningh 2007, 739 S., ISBN 978-3-506-75662-6
}

\footnotetext{
I Zwischen Alltag und Katastrophe. Der Dreißigjährige Krieg aus der Nähe, hg. von BENIGNA voN Krusenstjern, Hans Medick, in Zusammenarbeit mit PATRICE VEIT, Göttingen I999.
} 
Gemeinde- sowie Pfarrarchiven quer durch Süddeutschland. Die vielfach existenzielle Erfahrung der Kriegsläufte bewegte viele Menschen, das Erlebte schriftlich festzuhalten. Zwar sind ländliche und städtische Unterschichten und auch allgemein Frauen in den Zeugnissen unterrepräsentiert. Aber immerhin finden sich unter den Autoren neben Pfarrern auch zahlreiche Zunfthandwerker, Ackerbürger, Kaufleute, Ärzte und Apotheker, Bauern etc. Dies ist deswegen relevant, weil die ältere Forschung zum >Befreiungskrieg ausschließlich auf Stimmen aus dem Bildungsbürgertum rekurriert hatte. Das zentrale Frageinteresse der Arbeit richtet sich so auf die Wahrnehmungs- und Deutungsmuster der Zeitgenossen während der Kriege wie auch in der Verarbeitung des Geschehenen danach als Erinnerungskultur.

Die aufwändig recherchierte Nahperspektive auf das Kriegsgeschehen erbringt zahlreiche interessante Ergebnisse. Salopp formuliert, war die nationale Erhebung den meisten in Süddeutschland egal, wenn sie nicht der schmalen Elite der Honoratioren und Bildungsbürger angehörten. Allerdings gilt ebenso: "[A]uch wer nicht national dachte, weinte der napoleonischen Herrschaft kaum je eine Träne nach « (657). Viel wichtiger waren Probleme des Alltags, verursacht durch Plünderungen, Einquartierungen und Einberufungen. Bezeichnend ist die Praxis der Desertion. Diese folgte nicht einer Logik nationaler Affinität, sondern der Angst um das eigene Leben, egal gegen wen die Schlacht geschlagen wurde.

In mancher Hinsicht weisen die Praktiken und Wahrnehmungen während der Kriegszeit um I800 stärker zurück in die Frühe Neuzeit als voraus ins Zeitalter des Nationalismus und der bürgerlichen Gesellschaft. Dies gilt bereits für die Art und Weise der Kriegführung. Vor allem der Zwang zur Versorgung und zu sonstigen Hilfsleistungen, weniger die Gefechte und Schlachten, belastete die Zivilbevölkerung. Und wie schon während des Dreißigjährigen Krieges folgten Frauen und Kinder vielfach den Soldaten als ihren Partnern und Vätern in den Krieg. Die Kirchen - und mit ihnen zahlreiche Untertanen deuteten den Krieg zuerst als Strafe Gottes für sündiges Leben. Erst nach und nach wurden die Kirchen zu Agenturen eines neuen Territorialnationalismus in den Rheinbundstaaten. Eine intensivierte Frömmigkeit ist in beiden konfessionellen Lagern zu beobachten. Typisch blieb bis weit ins I9. Jahrhundert der Rückgriff auf »die gewohnten Methoden zur Kontingenzbewältigung " $(650)$ : Wallfahrten, Prozessionen, Gottesdienstbesuch. In pietistischen Konventikeln Württembergs zirkulierten Erwartungen auf das baldige Ende der Welt. Protestaktionen hatten meistens wie die zahlreichen Unruhen während der Frühen Neuzeit einen spezifisch lokalen Horizont. Das zukunftsorientierte Nations- und Freiheitskonzept der Französischen Revolution hatten die Akteurinnen und Akteure noch nicht übernommen. Ute Planert kann so zutreffend resümieren: »Überkommene Loyalitäten, Lokalismus und konfessionelle Haltungen bestimmten Weltanschauung und Handeln der meisten Menschen weit mehr als die moderne Idee der Nation « (659).

An manchen Stellen lässt sich fragen, ob dem preußisch-deutschen Nationalismus und seiner Deutung der Kriege gegen Frankreich als Negativfolie nicht zuviel Ehre und Aktualität zuteil wird. Hat nicht schon die Sozialgeschichte der I970er Jahre auf die Reformprozesse in den Rheinbundstaaten hingewiesen und die Preußenlastigkeit der älteren Forschung nachhaltig korrigiert? Wer spricht heute noch vom deutschen `Befreiungskrieg`? Aber ohne 
Zweifel ist Ute Planert ein äußerst profundes Werk gelungen, das unser Verständnis der 'Sattelzeit in vieler Hinsicht erweitert und ver- tieft. Das Buch hat eine breite Rezeption verdient.

Joachim Eibach

\section{Vom Untertan zum Citoyen*}

Die Französische Revolution ist bekanntlich ein fruchtbarer Gegenstand der Forschung. Auf dem von Skadi Krause bearbeiteten Feld kreuzen sich Forschungslinien sowohl der Politik- und der Rechtswissenschaft als auch der Rechtsgeschichte. Es geht um den Bruch im politischen Denken, der sich I788-I789 in den Debatten der Nationalversammlung vollzog. Durch die ganze Arbeit hindurch kann man feststellen, inwieweit Politik- und Rechtsverständnis im verfassungsgebenden Moment verknüpft waren. Anhand einer topisch gegliederten Untersuchung zeigt die Autorin pointiert, dass die Schaffung einer nationalen Repräsentation zur Delegitimierung monarchischer Herrschaft - so der Untertitel des Buches - beitrug. Zugleich spielten aber die herkömmlichen Leitbilder der Einheit und Unteilbarkeit der Souveränität und des politischen Willens eine wichtige Rolle bei der Etablierung der neuen politischen Ordnung. Die reiche, um den Begriff der nationalen Souveränität zentrierte Forschung weist auf diese Besonderheit der politischen Rhetorik der Französischen Revolution hin.

Die Arbeit orientiert sich an den großen Auseinandersetzungen der Zeit, die die Diskussion bestimmten. Zunächst steht der Schlüsselbegriff der Repräsentation der Nation im Mittelpunkt. Die Autorin zeigt, wie das tradierte Verständnis der Repräsentation in den Jahren I788 und I789 aufgegeben wurde. Dadurch wurden zwei herkömmliche Leitbilder in Frage gestellt. Zum einen wurde die Rolle des Königs als Repräsentanten der Nation durch die Rolle einer von der Person des Königs unabhängigen nationalen Repräsentation ersetzt. Zum anderen wurde die Bedeutung der Repräsentanten selbst umgedeutet. Da sie nicht mehr die Stände repräsentierten, sollten sie sich auch nicht mehr an Partikularinteressen orientieren. Die Auflösung der Generalstände zugunsten einer Nationalversammlung galt - und gilt immer noch - als Symbol dieser Neuorientierung. Hinzu kam die Abschaffung des imperativen Mandats. Erst hierdurch wurden die Abgeordneten fähig wie die Vertreter des Dritten Standes unterstrichen -, den allgemeinen Willen der Nation darzustellen. Diese Grundthesen werden von der Autorin durch zahlreiche Stellungnahmen von Abgeordneten illustriert, was zu begrüßen ist. Besonders die Übersetzung von wichtigen Teilen der Schrift von Emmanuel Joseph Sieyès "Qu'est-ce que le Tiers Etat « (35 f.) ist erhellend. Hier hätte aber die Bedeutung der Idee der Einheit der Nation in Frankreich - eine Auffassung, die mit der überlieferten Vorstellung von Souveränität verbunden war - noch mehr betont werden können. Diese Abstraktion bestimmt nämlich grundsätzlich und nach wie vor die Konstruktion des Staatsrechts in Frankreich. In diesem Sinne könnte der Verweis auf die berühmten Staatsrechtslehrer des Beginns des

\footnotetext{
* Skadi Krause, Die souveräne

Nation. Zur Delegitimierung monarchischer Herrschaft in Frankreich I 788-I789, Berlin: Duncker \& Humblot 2008, 248 S., ISBN 978-3-428-I 2733-7
} 